\title{
Plasma mediated neutrophil stimulation during coronary angioplasty: autocrine effect of platelet activating factor
}

\author{
Tomasz Siminiak, Daniel J O’Gorman, Manjit Shahi, David Hackett, Desmond J Sheridan
}

\begin{abstract}
Background-Polymorphonuclear neutrophils are involved in the development of myocardial injury during ischaemia and reperfusion. Coronary angioplasty has been shown to result in neutrophil activation. This may be a result of contact with ligands expressed by endothelial cells or response to soluble stimuli released from ischaemic tissue into the plasma or both.

Objective-To investigate plasma mediated neutrophil activation during angioplasty.
\end{abstract}

Methods and results-Plasma samples were collected from the coronary sinus, femoral artery, and femoral vein of 14 patients undergoing angioplasty, before and after the first balloon inflation and at the end of the procedure. Plasma samples were incubated with washed neutrophils isolated from healthy donors. Expression of the adhesion molecules CD18 integrin and L-selectin (Leu-8) was measured by flow cytometry, and superoxide anion production was measured by chemiluminescence. Plasma samples from the coronary sinus and femoral artery but not from the peripheral vein induced increased expression of neutrophil CD18 after balloon deflation. Modification of the expression of $L$-selectin was not noted. Production of superoxide anion by neutrophils was stimulated by plasma samples from the coronary sinus, but not by those from the femoral artery or vein. This plasma mediated neutrophil stimulation was prevented when the neutrophils were pretreated with platelet activating factor receptor antagonists BN52021 or BN50739. The platelet activating factor concentration detected in the coronary sinus was not higher than in control plasma.

Conclusion-Brief ischaemia during coronary angioplasty leads to the release of soluble stimuli capable of inducing neutrophil integrin expression and free oxygen radical production. Platelet activating factor may act as an autocrine neutrophil stimulus under these conditions.

(Br Heart $\mathcal{F} 1995 ; 74: 625-630)$

Keywords: neutrophil stimulation; platelet activating factor; coronary angioplasty; ischaemia/reperfusion

Substantial clinical and experimental evidence has been obtained in recent years to support the involvement of neutrophils in the development of myocardial injury, particularly during ischaemia followed by reperfusion. Neutrophil accumulation in the area of myocardial necrosis initiates a cascade of events resulting in the elimination of injured myocytes and subsequent scar formation. However, neutrophil activation in ischaemic myocardium may also result in the destruction of injured myocytes which have the potential for recovery. ${ }^{1}$

Activation of neutrophils during myocardial ischaemia/reperfusion may occur as a result of neutrophil contact with endothelial adhesion molecules expressed within ischaemic myocardium or in response to soluble stimuli released from ischaemic myocardium. Intravascular neutrophil activation results in the subsequent expression of surface adhesion molecules. Adhesion molecules expressed by activated neutrophils belong to the integrin family, of which the main molecule is $\mathrm{CD} 1 \mathrm{~b} / \mathrm{CD} 18$ and the selectin family, the most important of which is L-selectin. ${ }^{23}$

We have recently shown that plasma obtained from a peripheral vein in patients with acute myocardial infarction can induce chemotaxis, adherence, and superoxide anion production by neutrophils obtained from healthy control donors, ${ }^{4-6}$ suggesting release of neutrophilic stimuli from ischaemic myocardium into the peripheral circulation.

Several investigators have shown that ischaemia/reperfusion injury may be reduced by neutrophil depletion using a variety of methods including neutrophil antiserum, leukopak filters, or pharmacological interventions which modify neutrophil activation. ${ }^{1}$ Neutrophil induced biochemical damage of ischaemic myocardium may involve three mechanisms: (a) the release of free oxygen radicals, proteolytic enzymes, and leukotoxin by activated neutrophils $;^{78}$ (b) neutrophil activation in ischaemic myocardium results in their aggregation and adherence to endothelial cells with subsequent capillary plugging and impairment of coronary blood flow; ${ }^{7}$ and (c) vasoconstriction may result from leukotriene $\mathrm{B}_{4}$ release by activated neutrophils. ${ }^{9}$

The injurious role of neutrophil activation during myocardial infarction has been extensively studied, however, the role of neutrophil activation during brief periods of myocardial ischaemia, and its underlying mechanisms, remains unclear. Single reports indicate neutrophil activation in the coronary sinus after ischaemia caused by balloon inflation during coronary angioplasty. ${ }^{10-12}$ These findings suggest that myocardial ischaemia alone, without tissue necrosis, is sufficient to stimulate 

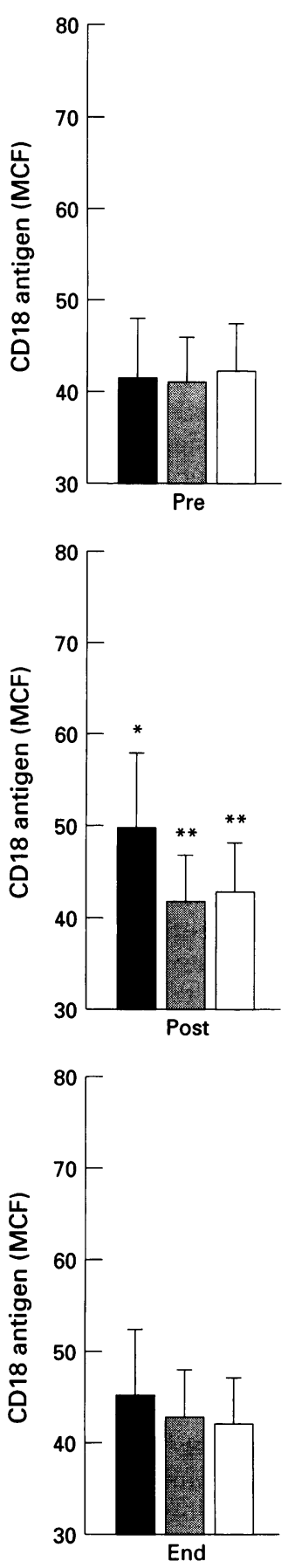

Figure 1 Expression of neutrophil CD18 antigen after incubation with plasma samples obtained from the coronary sinus before (pre) and after (post) the first balloon inflation and at the end of coronary angioplasty. Cells were pretreated with saline (घ) or platelet activating factor receptor antagonist BN52021 (훙) or BN50739 ( $\square$ ). Values are expressed as mean (SEM) mean channel of fluorescence (MCF).

$\star P<0.05$ versus preinflation; $\star \star P<0.05$ versus saline treated. neutrophils. Balloon dilatation induces arterial injury, and damage to endothelium can expose membrane receptors, for immunoglobin and complement factors, which promote neutrophil activation. ${ }^{13}$ Thus, although previous studies have shown neutrophil activation after coronary angioplasty, it is not known whether this stimulation occurs as a result of (a) the expression of endothelial receptors or (b) the release of soluble substances capable of neutrophil activation from ischaemic myocardium. As we have shown release of soluble stimuli for neutrophil activation after myocardial infarction, ${ }^{4-6}$ it may also be expected to occur after ischaemia induced by coronary angioplasty. Furthermore, platelet activating factor, a potent stimulus to neutrophil activation, has been shown to be released from ischaemic myocardium and may contribute to plasma mediated neutrophil stimulation after myocardial ischaemia. ${ }^{14-16}$ The purpose of the current study was to investigate whether substances capable of neutrophil activation are released during coronary angioplasty and whether platelet activating factor participates in this neutrophil stimulation. Coronary angioplasty is a model for brief reversible ischaemia. In order to exclude the possibility of systemic neutrophil activation secondary to prolonged myocardial ischaemia or necrosis, or both, we have excluded patients with unstable angina or recent myocardial infarction.

\section{Patients and methods}

PATIENTS

The study group included 14 patients undergoing angioplasty of the left anterior descending coronary artery. All patients had stable exertional angina and a significant stenosis of the proximal left arterior descending coronary artery. Patients with diabetes mellitus, renal failure, neoplastic conditions, or haematological diseases were excluded. No patient from the study group had suffered a previous myocardial infarction or undergone coronary bypass surgery. Patients with acute congestive heart failure or significant tricuspid regurgitation were also excluded. The patients' medications were continued on the day of the procedure. The medications were aspirin (150-300 $\mathrm{mg} /$ day in all patients), calcium channel blockers (seven patients), $\beta$ blockers (six patients), and long acting nitrates (four patients).

The study protocol was approved by the regional ethics committee.

\section{PROCEDURE}

After premedication with diazepam and local anaesthesia, an 8 French gauge sheath was placed in the femoral vein and a multipurpose catheter was advanced into the coronary sinus or, if not successful, as close to the sinus ostium as possible. An 8 French gauge sheath was placed in the femoral artery, the guiding catheter was introduced, and the coronary arteries were visualised. Isosorbide dinitrate (0.5 mg; Isoket, Schwarz Pharma, Chesham, Buckinghamshire) and 2000 units heparin were given by the intracoronary route and a further 8000 units were given intravenously. Blood samples were taken into EDTA containing tubes and after centrifugation plasma was stored at $-70^{\circ} \mathrm{C}$ until analysis. The initial samples were taken after administration of intracoronary drugs and contrast medium and before balloon inflation. A second set of samples were taken immediately after the first balloon deflation, and a final set at the end of the procedure-that is, $5 \mathrm{~min}$ after last deflation. Samples were taken from the coronary sinus ostium, femoral artery, and femoral vein. Angioplasty was performed with balloons ranging from 2.5 to $4.0 \mathrm{~mm}$ when inflated, chosen on the basis of the estimated diameter of normal segments adjacent to the stenosis. The duration of the first balloon inflation was prolonged to $2 \mathrm{~min}$, except in one patient in whom inflation was terminated after $90 \mathrm{~s}$ because of severe cardiac pain and pronounced ST segment elevation in the anterior leads. During the first inflation, 10 patients experienced moderate cardiac pain, 10 exhibited ST segment depression, and three had ST segment elevation in the anterior electrocardiogram leads. In total two to five inflations were performed in each patient to achieve a satisfactory result.

\section{NEUTROPHIL PREPARATION}

Peripheral blood neutrophils were isolated from EDTA samples obtained from healthy volunteers. Polymorphonuclear neutrophils were isolated by a single step centrifugation procedure on a Histopaque 1119/1077 (SigmaAldrich, Gillingham, Dorset) gradient, which is a modification of the original method described by Boyum. ${ }^{17}$ After isolation neutrophils were washed twice in phosphate buffered saline (Flow Laboratories, Rickmansworth, Hertfordshire) suspended to $10^{6} / \mathrm{ml}$ in Hanks' balanced salt solution (Sigma). Neutrophil suspensions were incubated with patient's plasma samples or autologous plasma in a ratio $1: 1$ for $20 \mathrm{~min}$ at $37^{\circ} \mathrm{C}$ after which neutrophil function was evaluated. In a further series of experiments neutrophils were preincubated for 10 min with platelet activating factor receptor antagonists BN 50739 or BN 52021 (Institut Henri Beaufour, Paris) in final concentrations of $1 \times 10^{-6} \mathrm{~mol} / \mathrm{l}$ and $1 \times 10^{-5} \mathrm{~mol} / \mathrm{l}$, respectively. These concentrations were proven sufficient to inhibit neutrophil activation by $10^{-9}-10^{-7} \mathrm{~mol} / 1$ platelet activating factor in vitro (data not shown). The in vitro effect of contrast medium used for visualisation of coronary arteries during angioplasty on neutrophil activation was evaluated by preincubation of the neutrophil suspension with the medium (Hexabrix320, May and Baker, Dagenham) diluted in autologous plasma to a final concentration of 10,20 , and $30 \%$.

\section{IMMUNOFLOW CYTOMETRY}

At the end of the incubation period equal numbers of cells were mixed with monoclonal antibodies to human cell surface receptors (CD18, CD11b, or Leu-8, Becton Dickinson, Oxford) together with $2 \%$ paraformaldehyde 

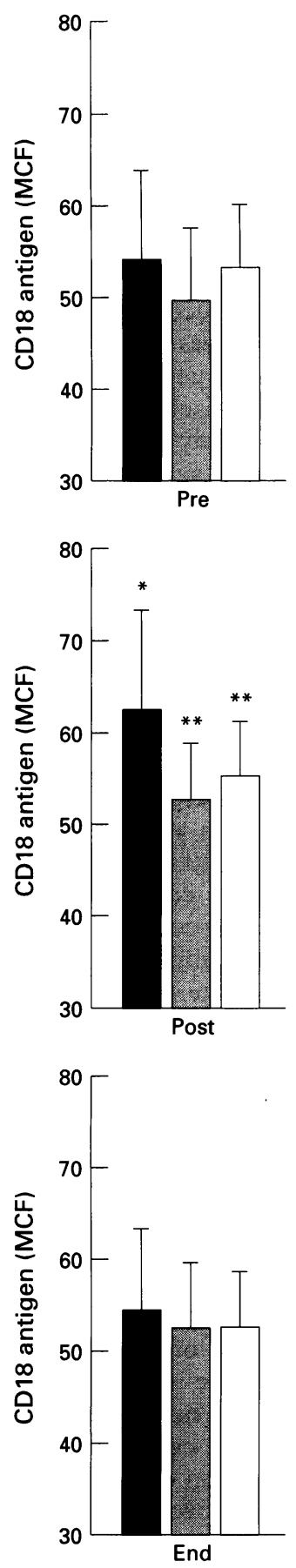

Figure 2 Expression of neutrophil CD18 antigen after incubation with plasma samples obtained from femoral artery before (pre) and after (post) the first balloon inflation and at the end of coronary angioplasty. Cells were pretreated with saline ( or platelet activating factor receptor antagonists BN52021 (i) or BN50739 ( $\square$ ). Values are expressed as mean (SEM) mean channel of fluorescence (MCF).

$\star P<0.05$ versus preinflation; $* \star P<0.05$ versus saline treated. fixative solution in phosphate buffered saline for $30 \mathrm{~min}$. Antibodies were conjugated with phycoerythrin or fluorescein. After three further washes cells were resuspended in Isoton (Becton Dickinson) for flow cytometry. Measurements were performed with a FACScan (Becton Dickinson) equipped with an argon laser (488 $\mathrm{nm}$ ) and three band pass filters. Data were analysed by gating the neutrophils forward scatter versus site scatter. Non-specific protein binding was assessed with an isotype matched immunoglobulin (IgG2b or IgG1, respectively; Becton Dickinson). Results are expressed as mean channel of fluorescence (relative units) calibrated by the equipment producer, as commonly used, ${ }^{18}$ and shown as the difference between specific and non-specific antibody binding.

\section{SUPEROXIDE ANION PRODUCTION}

Production of superoxide anion by neutrophils was measured using lucigenin amplified chemiluminescence according to a previously described method. ${ }^{19}$ Investigated or autologous plasma $(80 \mu \mathrm{l})$ was placed in each separated well of a white microtitre plate (Dynatech, Billinghurst, W Sussex) and neutrophil suspension $(80 \mu \mathrm{l})$ and lucigenin $(100 \mu \mathrm{l})$ at a concentration of $10^{-4} \mathrm{~mol} / \mathrm{l}$ in phosphate buffered saline were added. The measurements were performed in duplicate. Light emission was measured continuously for $60 \mathrm{~min}$ with the use of a microtitre plate based luminescence analyser (Amerlite, Kodak Clinical Diagnostics, Amersham, Buckinghamshire). The peak value detected under each well was recorded for statistical analysis. Results are expressed as a per cent of peak light emission of neutrophils incubated with autologous plasma.

PLATELET ACTIVATING FACTOR MEASUREMENT Samples were extracted according to LopezFarre et al. ${ }^{20}$ Briefly, methanol was added to serum or stimulated neutrophils in a ratio of 1.5:1 to extract lipid into the organic phase. Chloroform and water were then added to give a final ratio of methanol: water: chloroform of 1:0.9:1. The lower chloroform rich phase was aspirated and vacuum dried under nitrogen. Platelet activating factor was purified by fractionation on silica sample preparation cartridges. The dried samples were dissolved in 5 $\mathrm{ml}$ acidified chloroform ( $\mathrm{pH} 5$ ) and applied to activated $100 \mathrm{mg}$ silica cartridges (ISOLUTE, Jones Chromatography, Hengoed). The columns were serially eluted with $5 \mathrm{ml}$ chloroform: methanol (3:1), $5 \mathrm{ml}$ chloroform: methanol (3:2), $5 \mathrm{ml}$ chloroform: methanol (1:3) and platelet activating factor was eluted with $2 \mathrm{ml}$ methanol: water (3:1) into polypropylene tubes. This was again dried by vacuum under nitrogen and stored at $-70^{\circ} \mathrm{C}$ until analysis. Platelet activating factor was measured by a scintillation proximity radioimmuno assay (Amersham, Little Chalfont, Buckinghamshire). Samples were dissolved in assay buffer under the same conditions as standards.

\section{STATISTICAL METHODS}

Non-parametric statistics were used because our data were not normally distributed, as assessed by the Kolomogorov-Smirnov test. Evaluation was performed using the Friedmann multiple comparison paired test. Data are given as mean (SEM).

\section{Results}

CD18 antigen expression of control neutrophils incubated with plasma taken from the coronary sinus of patients before balloon inflation was $41.2(6 \cdot 6)$ (relative fluorescence units: mean channel of fluorescence) and significantly increased when incubated with plasma taken after the first balloon deflation (49.9 (7.9); $\mathrm{P}<0.05)$ but the difference was not significant when incubated with plasma taken the end of angioplasty procedure $(45 \cdot 0$ $(7 \cdot 1)$ ) (fig 1). Similarly, a significant increase in CD18 expression was observed after exposure of neutrophils to samples taken from the femoral artery after balloon deflation (62.6 $(10 \cdot 8))$ compared with arterial samples taken before inflation (54.2 (9.7); P < 0.05) but not to samples taken $5 \mathrm{~min}$ after last balloon deflation $(54 \cdot 6(8 \cdot 7)$ ) (fig 2 ).

Neutrophil integrin expression after incubation with samples obtained from femoral vein before angioplasty was $43 \cdot 2(5 \cdot 1)$ and was not significantly changed after the first balloon deflation $(46 \cdot 2(8 \cdot 1))$ or at the end of the procedure $(45 \cdot 1(7 \cdot 2))$, (fig 3$)$.

Pretreatment of neutrophils with the platelet activating factor receptor blocker BN52021 inhibited stimulation of CD18 expression by coronary sinus plasma after balloon deflation $(43.0(5.3) ; \quad P<0.05 v$ untreated neutrophils) and this effect was also observed with BN50739 (41.9 (4.9)); P < $0.05 v$ untreated neutrophils) (fig 1). Similarly, stimulation of CD18 expression by arterial plasma taken after balloon deflation was not observed in the presence of BN52021 (55.5 (5.8); $\quad \mathrm{P}<0.05 \quad v$ untreated) or BN50739 (52.8 (6.1)); P < 0.05).

The expression of L-selectin was not affected by incubation with plasma obtained after balloon deflation from the coronary sinus (before inflation $12.8(4.0) v 10.5(3.2)$ postdeflation), femoral artery $(11.9(4 \cdot 2) v 11.2$ $(4 \cdot 4)$, or femoral vein $(13.4(6 \cdot 1) v 11 \cdot 8(4 \cdot 9))$.

Superoxide anion production by neutrophils (expressed as per cent of production after incubation with autologous plasma) after incubation with plasma from the coronary sinus before balloon inflation was $92 \cdot 1(5 \cdot 8) \%$ and increased after the first balloon deflation to $121.2(6.1) \% \quad(P<0.05)$ but was not increased when incubated with plasma taken at the end of the angioplasty procedure (94.4 $(6 \cdot 2) \%$ ) (fig 4). Plasma mediated stimulation of neutrophil superoxide anion production was reduced in the presence of BN52021 (101.9 (2.9)\%; $\mathrm{P}<0.05 v$ untreated) or BN50739 (103.9 (3.8)\%; P < 0.05). No significant difference in superoxide production was observed when neutrophils were incubated with plasma taken from the femoral 

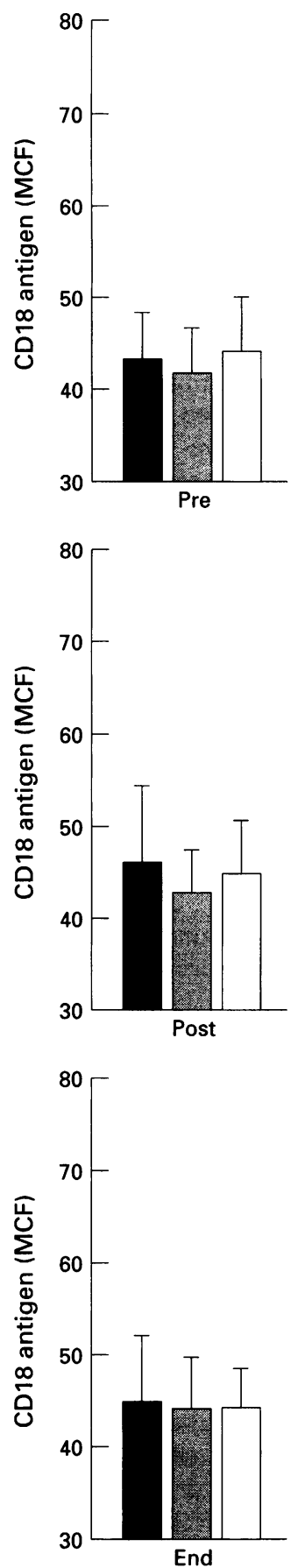

Figure 3 Expression of neutrophil CD18 antigen after incubation with plasma samples obtained from femoral vein before (pre) and after (post) the first balloon inflation and at the end of coronary angioplasty. Cells were pretreated with saline (D) or platelet activating factor receptor antagonists BN52021 (늘 ) or BN50739 ( $\square$ ). Values are expressed as mean (SEM) mean channel of fluorescence (MCF). artery $(101 \cdot 2(5 \cdot 8), 108 \cdot 7(4 \cdot 8)$, and $104 \cdot 2$ $(5 \cdot 1) \%$ for samples taken before and after the first balloon inflation and at the end of the procedure, respectively) or femoral vein $(98.2$ $(4 \cdot 9), 97 \cdot 3(5 \cdot 1)$, and $101 \cdot 0(6 \cdot 9) \%)$.

In vitro incubation of neutrophils with contrast medium diluted to concentrations of 10 , 20 and $30 \%$ in autologous plasma did not alter CD18 expression $(44 \cdot 1(5 \cdot 1), 43 \cdot 8(4 \cdot 2)$, and $44 \cdot 0(6 \cdot 1)$, respectively), L-selectin expression $(12 \cdot 1(4 \cdot 2), 11.9(5 \cdot 1)$, and $10.9(3 \cdot 8))$, or superoxide anion production $(102 \cdot 3(2 \cdot 1)$, $101 \cdot 2(2 \cdot 0)$, and $100 \cdot 9(2 \cdot 9) \%)$.

Concentrations of platelet activating factor in the coronary sinus plasma samples taken after the first balloon inflation were not higher than in samples taken before angioplasty (post-deflation $3.5(0.5) \quad v \quad 3.1(0.4) \mathrm{ng} / \mathrm{ml}$ before inflation) or in venous plasma from healthy controls $(3 \cdot 0(0 \cdot 4) \mathrm{ng} / \mathrm{ml})$.

\section{Discussion}

The pathophysiological role of activated neutrophils in the development of myocardial injury during ischaemia/reperfusion is related to their adherence to endothelial cells with subsequent coronary capillary plugging and reduction in coronary blood flow, as well as to the release of highly reactive oxygen species which exert a cytotoxic effect on injured myocytes, which would otherwise have the potential to recover. ${ }^{1}$

The adhesion of neutrophils to endothelial cells requires the expression of adhesion molecules on the endothelial cell and neutrophil. Neutrophils constitutively express the $\beta_{2}$ integrin family of adhesion molecules. These glycoproteins contain a common $\beta$ chain (CD18) and a distinct $a$ subunit (CD11a, CD11b, CD11c), and react with intercellular adhesion molecules- 1 and -2 expressed by endothelial cells (reviewed in reference 2). Furthermore, neutrophils express L-selectin, a member of the selectin family of glycoproteins, which interacts with sialyl Lewis ${ }^{x}$ antigen expressed by activated endothelial cells, and probably with other, unidentified endothelial counter receptors.

Several investigators have confirmed the involvement of the $\beta_{2}$ integrin family in increased neutrophil adherence to endothelium during myocardial ischaemia. Increased expression of the CD11b/CD18 complex was shown in neutrophils taken from the coronary sinus of patients with unstable angina compared with that of neutrophils taken from the aorta, indicating a transcardiac increase of neutrophil adherence molecules as a result of myocardial ischaemia. ${ }^{21}$ In addition, administration of $\mathrm{F}\left(\mathrm{ab}^{\prime}\right)_{2}$ fragments of a monoclonal antibody against the CD18 chain inhibited neutrophil accumulation in the myocardium after experimental infarction followed by reperfusion, ${ }^{56}$ as did antibodies that recognise the CD11b $a$ subunit. ${ }^{22} \mathrm{~A}$ significant increase in coronary blood flow was observed in an experimental ischaemia/reperfusion model after administration of antibodies that blocked $\mathrm{CD} 18^{23}$ or $\mathrm{CD} 11 \mathrm{~b}$ subunits. ${ }^{24}$ Thus, the expression of neutrophil adhesion molecules may be involved in capillary plugging and contribute to the no reflow phenomenon.

Previous studies have indicated that Lselectin, which is thought to have a role in neutrophil "rolling along" the endothelium, is involved in the extravasation and subsequent accumulation of neutrophils in ischaemic myocardium. ${ }^{3}$ Administration of a monoclonal antibody that recognises L-selectin attenuated myocardial necrosis and decreased neutrophil accumulation in ischaemic myocardium in a feline model of experimental ischaemia/reperfusion. ${ }^{25}$ Furthermore, antibody against neutrophilic L-selectin protected against the loss of endothelium dependent relaxation observed in coronary artery rings isolated from cats subjected to myocardial ischaemia and reperfusion. The contribution of P-selectin complexes, present on activated endothelium, to neutrophil adhesion has been suggested from studies utilising a monoclonal antibody to P-selectin. ${ }^{26} 27$

This study provides direct evidence that brief periods of myocardial ischaemia during coronary angioplasty result in the release of soluble factors capable of stimulating neutrophil expression of CD18, a common chain of the integrin family of adhesion molecules. These can be detected in samples from the coronary sinus and femoral artery. Neutrophil activation has previously been shown to occur as a result of coronary angioplasty. ${ }^{1028}$ Increased plasma levels of neutrophil elastase have been detected in the coronary sinus after angioplasty. ${ }^{10}$ Neutrophils released into the coronary sinus after balloon deflation were shown to be activated, as assessed by increased nitro-blue tetrazolium reduction and decreased filterability. ${ }^{12}$ It has been suggested that neutrophil activation during coronary angioplasty may be an effect of the contrast medium. ${ }^{18}$ However, we have not observed any significant modification of neutrophil activation after incubation of the cells with contrast medium at a concentration of $10-30 \%$ in autologous plasma. Furthermore, the first set of samples in each patient was taken after coronary artery visualisation and intracoronary drug administration, immediately before balloon inflation, and thus included possible effects due to drugs or contrast media.

Neutrophil activation after coronary angioplasty may be the result of direct cell contact with endothelial adhesion molecules or stimulation by soluble neutrophilic stimuli released from ischaemic myocardium, or both. The results from our current study provide evidence for plasma mediated neutrophil stimulation during angioplasty. Immediately after the first balloon deflation plasma samples obtained from the coronary sinus and the femoral artery contained factors capable of inducing CD18 antigen expression on the surface of washed neutrophils from healthy donors and coronary sinus plasma was capable of stimulating superoxide anion production by donor neutrophils. This effect has not been observed at the end of the procedure suggesting a rapid washout of soluble factors from reper- 

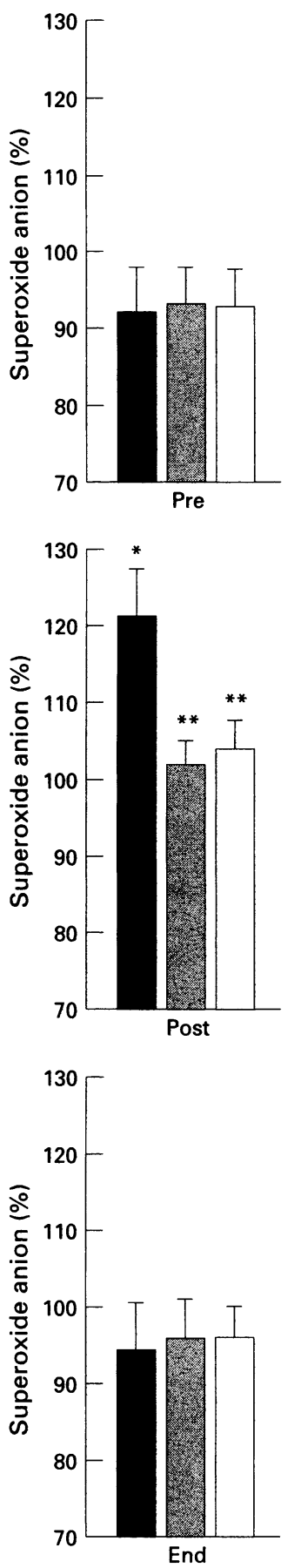

Figure 4 Neutrophil superoxide anion production after incubation with plasma samples obtained from the coronary sinus before (pre) and after (post) the first balloon inflation and at the end of coronary angioplasty. Cells were pretreated with saline (1) or platelet activating factor receptor antagonists

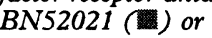
BN50739 ( $\square$ ). Values are expressed as mean (SEM) per cent of production after incubation with autologous plasma. ${ }^{\star} P<0.05$ versus preinflation; $* * P<0.05$ versus saline treated. fused myocardium. These findings are consistent with a previous report by Neumann et al, ${ }^{12}$ who detected release of neutrophilic chemoattractants into the coronary sinus, which were also capable of stimulating the release of neutrophil superoxide anion as measured by a reduction in cytochrome c. Intracardiac activation of neutrophil respiratory bursts was indicated by observations that neutrophils obtained from the coronary sinus released less superoxide anion in response to phorbolmyristate-acetate stimulation. ${ }^{10}$

Deep entry into the coronary sinus was not possible in all our patients, resulting in some contamination with systemic venous blood. There was no increase in neutrophil activating stimuli in samples taken from the femoral vein (fig 3), implying that the increase in activity in the coronary sinus has been underestimated.

The nature of the neutrophilic stimuli released during coronary angioplasty is uncertain. In the present study, pretreatment of donor neutrophils with platelet activating factor receptor blockers BN52021 and BN50739 decreased plasma mediated stimulation. Platelet activating factor is a potent stimulus for neutrophil activation ${ }^{10}$ and has been shown to be released by ischaemic endothelial cells resulting in greater adherence of the neutrophils to the endothelium. ${ }^{15}$ Conflicting results have been reported concerning the role of platelet activating factor as a neutrophil stimulus during ischaemia/reperfusion. Some reports indicate that neutrophil dependent tissue injury during myocardial ischaemia is, in part, related to activation by platelet activating factor, ${ }^{29}$ whereas in other studies administration of a platelet activating factor antagonist had no effect on neutrophil accumulation after ischaemic/reperfusion injury. ${ }^{30}$ Similarly, conflicting results have been reported on the release of platelet activating factor into the coronary sinus after brief myocardial ischaemia. The level of platelet activating factor has been shown to be increased in the great cardiac vein during coronary angioplasty, ${ }^{31}$ whereas others have not detected increased concentrations of platelet activating factor in the coronary sinus during angioplasty nor after rapid atrial pacing in patients with ischaemic heart disease despite evidence of ischaemia defined by lactate extraction. ${ }^{32}$ In this study platelet activating factor concentrations in samples capable of activating neutrophils were not increased during coronary angioplasty, suggesting that platelet activating factor released from ischaemic myocardium is not directly involved in plasma mediated neutrophil stimulation.

Neutrophils themselves are a rich source of platelet activating factor ${ }^{33}$ and recent studies suggest that platelet activating factor is an important mediator of neutrophil-platelet cooperation in ischaemic myocardium. ${ }^{34}$ Mechanical and electrical dysfunction caused by platelet infusion in isolated hearts during ischaemia/reperfusion was significantly worsened in the presence of neutrophils, and this effect was prevented by treatment with a platelet activating factor receptor antagonist. ${ }^{35}$
Platelet activating factor released by activated neutrophils may also act as an autocrine stimulus. Exposure of neutrophils to certain stimuli, for example endothelin, results in platelet activating factor production by neutrophils and subsequent autocrine stimulation. This explains why cell pretreatment with platelet activating factor receptor blockers inhibits platelet activating factor mediated neutrophil self-stimulation. ${ }^{14}$ In the present study the finding that platelet activating factor receptor blockade inhibited plasma mediated neutrophil stimulation after angioplasty in the absence of increased plasma levels of platelet activating factor suggests that it acts as an autocrine neutrophil stimulus-that is soluble stimuli released from ischaemic myocardium during angioplasty activate neutrophils, which subsequently release platelet activating factor inducing further self-stimulation of neutrophils.

The pathophysiological importance and clinical relevance of neutrophil activation during myocardial infarction are accepted. The importance of neutrophil activation during brief ischaemia without necrosis, however, remains unclear. Neutrophil activation during brief ischaemia results in the release of oxygen free radicals and could contribute to myocardial stunning, ${ }^{35}$ although recent data have failed to support the role of neutrophil activation in the decrease of contractile function in stunned myocardium. ${ }^{36}$

Pronounced alterations in diastolic function are also associated with coronary angioplasty. ${ }^{37}$ It can therefore be speculated that activated neutrophils may contribute to this process. The present findings support the concept of plasma mediated neutrophil stimulation during coronary angioplasty and confirm the usefulness of angioplasty as a model of brief myocardial ischaemia in man. Future research on the nature and mechanisms of neutrophil activation may infer a pharmacological approach to minimise neutrophil mediated myocardial injury during ischaemia.

1 Siminiak T, Ozawa T. Neutrophil mediated myocardial injury. Int f Biochem 1993;25:147-56.

2 Zimmermann GA, Prescott SM, McIntyre TM. Endothelial interactions with granulocytes: tethering and signaling molecules. Immunol Today 1992;13:93-100.

3 Lefer AM, Weyrich AS, Buerke M. Role of selectins, a new family of adhesion molecules in ischaemia-reperfusion injury. Cardiovasc Res 1994;28:289-94.

4 Siminiak T, Zozulinska D, Zeromska M, Wysocki $H$. Evidence for plasma mediated neutrophil superoxide anion production during myocardial infarction. Cardiology 1993;82:377-82.

5 Klimas R, Wysocki H, Siminiak T. Streptokinase modifies neutrophil adherence-augmenting and chemotaxisinducing plasma activity. Thromb Haemost 1991;65(6): 1240.

6 Siminiak T, Wasiewicz A, Klimas R, Kazmierczak M, Wysocki H. Streptokinase treatment affects neutrophil aggregation. Eur $\mathcal{F}$ Pharmacol 1990;183:1845.

7 Engler RL. Consequences of activation and adenosinemediated inhibition of granulocytes during myocardial schemia. Fed Proc 1987;46:2407-12.

8 Bell D, Jackson M, Nicoll J, Millar A, Daws J, Muir AL. Inflammatory response, neutrophil activation and free radical production after acute myocardial infarction: effect of thrombolytic treatment. Br Heart $\mathcal{f}$ 1990;63: 82-7.

9 Mullane KM, Hatala MA, Kraemer R, Sessa W, Westlin W. Myocardial salvage induced by REV-5901: an inhibitor and antagonist of leukotrienes. $\mathcal{f}$ Cardiovasc Pharmacol 1987;10:398-406.

10 De Servi S, Mazzone A, Ricevutti G, Fioravanti A, Bramucci E, Angoli L, et al. Granulocyte activation after coronary angioplasty in humans. Circulation 1990;82: $140-6$. 
11 Rauch B, Neumann J, Richardt G, Kranzhofer R, Barth R, Zimmermann $\mathrm{R}$, et al. Effect of gallopamil on myocardial ischaemia during percutaneous transluminal coronar angioplasty. Drugs 1991;42(suppl 1):31-6.

12 Neumann F-J, Richardt G, Schneider M, Ott I, Haupt $\mathrm{H}-\mathrm{M}$, Tillmanns $\mathrm{H}$, et al. Cardiac release of chemoattractants after ischaemia induced by coronary balloon angioplasty. Br Heart f 1993;70:27-34.

13 Mayrovitz $\mathrm{H}$, Wiedemann $M$, Tuma $\mathrm{R}$. Factors influencing leukocyte adherence in microvessels. Thromb Haemost 1977;38:823-31.

14 Gomez-Garre D, Guerra M, Gonzales E, Lopez-Farre A, Riesco A, Caramelo C, et al. Aggregation of human polymorphonclear leukocytes by endothelin: role of plateletmorphonuclear leukocytes by endothelin: role of plate

15 Milhoan KA, Lane TA, Bloor CM. Hypoxia induces endothelial cells to increase their adherence for endothelial cells to increase their adherence for
neutrophils: role of PAF. Am $f$ Physiol 1992;263: neutrophils:

16 Vercellotti GM, Yin HQ, Gustafson KS, Nelson RD, Jacob HS. Platelet-activating factor primes neutroph responses to agonists: role in promoting neutrophil-medated endothelial cell damage. Blood 1988;71:1100-7.

17 Boyum A. Isolation of mononuclear cells and granulocyte from human blood. Scand F Clin Lab Invest 1968;21 77-89.

18 Feldman L, Chollet-Martin S, Himbert D, Juliard JM, Pasquier C, Elbim C, et al. Does PTCA really activate granulocytes? Role of contrast media [abstract] Circulation 1993;88(4):I-338.

19 Siminiak T, Wysocki H, Veit A, Maurer HR. The effect of selected antiarrhythmic drugs on neutrophil free oxygen radicals production measured by chemiluminescence. radicals production measured by
Basic Res Cardiol 1991;86:355-62.

20 Lopez-Farre A, Torralbo M, Lopez-Novoa JM. Glomerul from ischaemic rat kidneys produce increased amounts of from ischaemic rat kidneys produce increased amounts of platelet activating

21 Ricevuti G, Mazzone A, Mazzucchelli I, Fossati G, Pasott $\mathrm{D}$, Cavigliano $\mathrm{P}$, et al. Phagocyte activation in coronary artery disease. FEMS Microbiol Immunol 1992:105:271-8.

22 Simpson PJ, Todd RF, Fantone JC, Mickelson JK, Griffin JD, Lucchesi BR. Reduction of experimental canine myocardial reperfusion injury by a monoclonal antibody (anti-Mol, anti-CD11b) that inhibits leukocyte adhesion $\mathcal{f}$ Clin Invest 1988;81:624-9.

23 Tanaka M, Brooks SE, Richard VJ, FitzHarris GP, Stoler RC, Jennings RB, et al. Effect of anti-CD18 antibody on myocardial neutrophil accumulation and infarct size after ischaemia and reperfusion in dogs. Circulation 1993, 87:526.

24 Simpson PI, Todd RF III, Mickelson JK, Fantone JC Gallager KP, Lee KA, et al Sustained limitation, myocardial reperfusion injury by a monoclonal antibody that alters leukocyte function. Circulation 1990;81: 226-37.
$25 \mathrm{Ma} \mathrm{X}$, Weyrich AS, Lefer DJ, Buerke M, Albertine KH, Kishimoto TK, et al. Monoclonal antibody to L-selectin attenuates neutrophil accumulation and protects ischaemic reperfused cat myocardium. Circulation 1993; 88:649-58.

26 Lefer DJ, Serrano CV, Shandelya SML, Sterinsky SA, Bochner BS, Zweier JL. A novel anti-P-selectin monoclonal antibody (MAb) attenuates neutrophil mediated postischemic myocardial contractile dysfunction [abstract]. Circulation 1993;88(suppl I):I-443.

27 Hendricks JB, Chen LY, Yang BC, Nichols WW, Mehta IL. A novel anti-P-selectin monoclonal antibody (MAb) attenuates neutrophil mediated postischemic myocardial contractile dysfunction [abstract]. Circulation 1993; 88(suppl I):I-443.

28 Neumann FJ, Ott I, Richardt G, Zimmermann R, Tillmanns $\mathrm{H}$, Kubler $\mathrm{W}$, et al. Effect of gallopamil on
neutrophil functions: experimental and clinical studies. $\mathcal{f}$ neutrophil functions: experimental and clinical

29 Stahl GL, Terashita Z, Lefer AM. Role of platelet activating factor in myocardial ischaemia in rats. Circ Shock 987;21:298-301.

30 Williams FM, Collins PD, Tanniere-Zeller M, Williams TJ. The relationship between neutrophils and increased microvascular permeability in a model of myocardial ischaemia and reperfusion. Br f Pharmacol 1990;100: 729-34

31 Eldar M, Lysko PG, Schulhoff N, Gagnon RC, Feuerstein G, Shani J. Effects of coronary angioplasty on plasma platelet-activating factor in man. $\mathcal{F}$ Lipid Mediat 1992; 5:313-9.

32 Montalescot G, Maclouf J, Drobinski G, Mencia-Huerta JM, Ankri A, Grosgogeat Y, et al. Lack of platelet-activating factor release during reversible myocardial ischaemia. Eur Heart 7 1992;13:1641-4.

33 Weksler BB. Platelets. In: Gallin II, Goldstein IM, Snyderman R, eds. Inflammation: basic principles and Snyderman R, eds. Inflammation: basic principles and

34 Alloattii G, Montrucchio G, Emanuelli G, Camussi G. Platelet-activating factor (PAF) induces platelet/neutrophil co-operation during myocardial reperfusion. $f$ Mol Cell Cardiol 1992;24:163-71.

35 Engler RL, Covell JW. Granulocytes cause reperfusion ventricular dysfunction after $15 \mathrm{~min}$ ischaemia in dog. Circ Res 1987;61:20-8.

36 Juneau CF, Ito BR, del Balzo U, Engler R. Severe neutrophil depletion by leucocyte filters or cytotoxic drug does not improve recovery of contractile function in stunned porcine myocardium. Cardiovasc Res 1993;27: 720-7.

37 Wijns W, Serruys PW, Slager CL, Grimm J, Krayenbuehl HP, Hugenholtz PG, et al. Effect of coronary occlusion during percutaneous transluminal angioplasty in humans during percutaneous transluminal angioplasty in humans on left ventricular chamber stiffness and regional dias455-63. 